\title{
Interference-Aware IEEE 802.16 WiMax Mesh Networks
}

\author{
Hung-Yu Wei, Samrat Ganguly, Rauf Izmailov \\ Broadband \& Mobile Networking Department \\ NEC Laboratories America \\ Princeton, New Jersey, USA \\ \{hungyu, samrat, rauf\}@nec-labs.com
}

\author{
Zygmunt J. Haas \\ School of Electrical and Computer Engineering \\ Cornell University \\ Ithaca, New York, USA \\ haas@ece.cornell.edu
}

\begin{abstract}
The IEEE 802.16 WiMax standard provides a mechanism for creating multi-hop mesh, which can be deployed as a high speed wide-area wireless network. To realize the full potential of such high-speed IEEE 802.16 mesh networks, two efficient wireless radio resource allocation extensions were developed. The objective of this paper is to propose an efficient approach for increasing the utilization of WiMax mesh through appropriate design of multi-hop routing and scheduling. As multiple-access interference is a major limiting factor for wireless communication systems, we adopt here an interference-aware cross-layer design to increase the throughput of the wireless mesh network. In particular, our scheme creates a tree-based routing framework, which along with scheduling is interference aware and results in a much higher spectral efficiency. Performance evaluation results show that the proposed interference-aware scheme achieves significant throughput enhancement over the basic IEEE 802.16 mesh network.
\end{abstract}

Keywords-IEEE 802.16, Mesh Network, WiMax, Cross-layer Design and Optimization, Multi-hop Routing, Mesh Mode

\section{INTRODUCTION}

The IEEE 802.16 Working Group created a new standard, commonly known as WiMax, for broadband wireless access at high speed, at low cost, which is easy to deploy, and which provides a scalable solution for extension of a fiber-optic backbone. WiMax base stations can offer greater wireless coverage of about 5 miles, with LOS (line of sight) transmission within bandwidth of up to $70 \mathrm{Mbps}$.

Beyond just providing a single last hop access to a broadband ISP, WiMax technology can be used for creating wide-area wireless backhaul network. When a backhaul-based WiMax is deployed in Mesh mode, it does not only increase the wireless coverage, but it also provides features such as lower backhaul deployment cost, rapid deployment, and reconfigurability. Various deployment scenarios include citywide wireless coverage, backhaul for connecting $3 \mathrm{G} \mathrm{RNC}$ with base stations, and others.

In addition to the single-hop IEEE 802.16 PMP (point-tomultipoint) operation, IEEE 802.16a standard [1] defined the basic signaling flows and message formats to establish a mesh network connection. Subsequently, the Mesh mode specifications were integrated into the IEEE 802.16-2004 revision [2]. Although single hop WiMax provides high flexibility to attain Quality of Service in terms of data throughput, achieving the same in multi-hop WiMax mesh is challenging. One of the major problem is dealing with the interference from transmission of the neighboring WiMax nodes.

Cross-layer design and optimization is known to improve the performance of wireless communication and mobile networks [3,4]. In order to design a spectrally efficient IEEE 802.16 mesh network, we opt for joint design and optimization that relies on Application Layer load demand information, Physical Layer interference information, as well as scheduling and route selection mechanism in Data Link Layer. We believe that interference in wireless systems is one of the most significant factors that limit the network capacity and scalability of wireless mesh networks. Consideration of interference conditions during radio resource allocation and route formation processes impacts the design of concurrent transmission schemes with better spectral utilization while limiting the mutual interference.

We developed an interference-aware IEEE 802.16 framework with a design goal of achieving overall high utilization of the WiMax Mesh network. Our proposed scheme includes a novel interference-aware route construction algorithm and an enhanced centralized mesh scheduling scheme, which consider both traffic load demand and interference conditions. This interference-aware design approach will lead to better spatial reuse and thus higher spectral efficiency.

The rest of the paper is organized as follows: in Section II, we briefly overview the IEEE 802.16 Mesh mode operation. In Section III, we present the interference-aware framework that includes route construction and scheduling algorithm. In Section IV, the IEEE 802.16 mesh network performance is evaluated via simulations and compared to theoretical upper bound, which we found using linear programming. Finally, we summarize our results and provide some concluding remarks in Section V. 


\section{OVERVIEW OF WiMAX MeSH}

\section{A. Motivation and Problem Overview}

In comparison to IEEE $802.11 \mathrm{a} / \mathrm{b} / \mathrm{g}$ based mesh network, the 802.16-based WiMax mesh provides various advantages apart from increased range and higher bandwidth. The TDMA based scheduling of channel access in WiMax-based multi-hop relay system provides fine granularity radio resource control, as compared to RTS/CTS-based $802.11 \mathrm{a} / \mathrm{b} / \mathrm{g}$ systems. This TDMA based scheduling mechanism allows centralized slot allocation, which provides overall efficient resource utilization suitable for fixed wireless backhaul network. (The WiMaxbased mesh backhaul application differs from the $802.11 \mathrm{a} / \mathrm{b} / \mathrm{g}$ based mesh, which targets mobile ad hoc networks.) However, the interference remains a major issue in multi-hop WiMax mesh networks. To provide high spectral usage, an efficient algorithm for slot allocation is needed, so as to maximize the concurrent transmissions of data in the mesh. The level of interference depends upon how the data is routed in the WiMax network.

In this paper, we consider the following scenario of WiMax-based mesh deployment. A mesh network is managed by a single node, which we refer to as Mesh BS. Mesh BS serves as the interface for WiMax-based mesh to the external network. We provide an algorithm for interference-aware multi-hop route selection for a given capacity-request matrix, which leads to efficient scheduling.

TABLE I. IEEE 802.16 MESH MODE ACRONYMS

\begin{tabular}{|l|c|}
\hline BS & Base Station \\
\hline SS & Subscriber Station \\
\hline MSH & Mesh \\
\hline SN & Sponsoring Node \\
\hline CN & Candidate Node \\
\hline MSH-NCFG & Mesh network Configuration Message \\
\hline MSH-NENT & Mesh Network Entry Message \\
\hline MSH-CSCH & Mesh Centralized Scheduling Message \\
\hline MSH-CSCF & Mesh Centralized Scheduling Configuration Message \\
\hline
\end{tabular}

\section{B. IEEE 802.16 Mesh Mode Operations}

In IEEE 802.16 Mesh mode, a Mesh base station (BS) provides backhaul connectivity of the mesh network and controls one or more subscriber stations (SS). When centralized scheduling scheme is used, the Mesh BS is responsible for collecting bandwidth request from subscriber stations and for managing resource allocation. We will first introduce the 802.16 Mesh network entry process (i.e., a process by which a new node joins the mesh), and then we describe the network resource allocation request/granting procedure.

In IEEE 802.16 Mesh mode, Mesh Network Configuration $(M S H-N C F G)$ and Mesh Network Entry (MSH-NENT) messages are used for advertisement of the mesh network and for helping new nodes to synchronize and to joining the mesh network. Active nodes within the mesh periodically advertise MSH-NCFG messages with Network Descriptor, which outlines the basic network configuration information such as BS ID number and the base channel currently used. A new node that plans to join an active mesh network scans for active networks and listens to MSH-NCFG message. The new node establishes coarse synchronization and starts the network entry process based on the information given by MSH-NCFG. Among all possible neighbors that advertise MSH-NCFG, the joining node (which is called Candidate Node in the 802.16 Mesh mode terminology) selects a potential Sponsoring Node to connect to. A Mesh Network Entry message (MSH-NENT) with NetEntryRequest information is then sent by the Candidate Node to join the mesh.

The IEEE 802.16 Mesh mode MAC supports both centralized scheduling and distributed scheduling. Here, we focus on the centralized mesh scheme to establish high-speed broadband mesh connections, where the Mesh BS coordinates the radio resource allocation within the mesh network. In the centralized scheme, every Mesh SS estimates and sends its resource request to the Mesh BS, and the Mesh BS determined the amount of granted resources for each link and communicates. The request and grant process uses the Mesh Centralized Scheduling (MSH-CSCH) message type. A Subscriber Stations capacity requests are sent using the $M S H$ CSCH:Request message to the Subscriber Station's parent node. After the Mesh BS determines the resource allocation results, the MSH-CSCH:Grant is propagated along the route from Mesh BS. To disseminate the link, node, and scheduling tree configuration information to all participants within the mesh network, the Mesh Centralized Scheduling Configuration (MSH-CSCF) message is broadcasted by the Mesh BS and then re-broadcasted by intermediate nodes.

\section{INTERFERNCE-AWARE DESIGN WITH 802.16 MESH}

\section{A. Interference-Aware Route Construction}

To achieve efficient spectral utilization and high throughput in 802.16 mesh networks, the route construction within the mesh network is crucial. To this end, we propose an interference-aware route construction algorithm that considers interference condition in the mesh network. The concept of blocking metric $B(k)$ of a given route from the Mesh BS toward an SS node $k$ is introduced to model the interference level of routes in the mesh.

The blocking metric $B(k)$ of a multihop route indicates the number of blocked (interfered with) nodes by all the intermediate nodes along the route from the root node toward the destination node $k$. We also define the blocking value $\mathrm{b}(\eta)$ of a node $\eta$, as the number of blocked (interfered with) nodes when node $\eta$ is transmitting. Therefore, the blocking metric of a route will be the summation of the blocking values of nodes that transmit or forward packets along the route. A simple example of blocking metric computation is shown in Figure 1. In this example, a node is blocked when it is within the transmission range of the transmitting node. Thus, node $\eta_{i}$ 's 
blocking value $\mathrm{b}\left(\eta_{i}\right)$ equals the number of neighboring nodes of $\eta_{i}$. The blocking metric along a route toward $k, B(k)$ is equal to the summation of all the blocking value $\mathrm{b}\left(\eta_{i}\right)$ for every node $\eta_{i}$ (including the source node and all the forwarding nodes) on the route $k$. In the example in Figure 1, $B(k)$ is computed by adding $b(s), b(n 1), b(n 2)$, and $b(n 3)$.

As shown in Figure 1, nodes are blocked if their transmissions would interfere with the currently receiving node. Similarly, Figure 2 shows an example of blocking metric computation of a route with larger interference than the example in Figure 1. Our design approach in the proposed interference-aware scheme is to select the routes with less interference. For the clarity of illustration in Figure 1 and 2, the blocking metric computation presented here shows a simplified case, where only nodes within the transmission range of a transmitting node are blocked. In various scenarios, a transmitting node could interfere with nodes that are in a larger distance away. Other types of blocking metrics (such as detailed propagation model or measurement with receiver sensitivity) could be defined based on the information availability and system design tradeoffs.

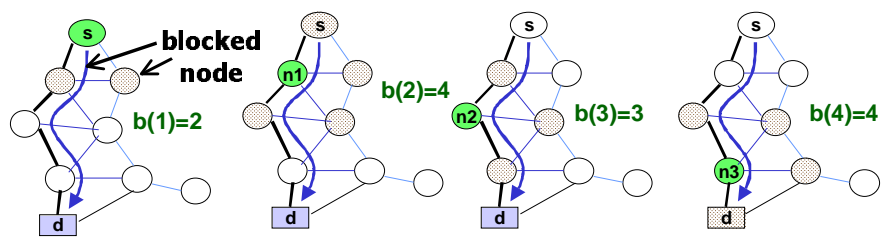

Figure 1. An exmple of blocking metric $B(k)=2+4+3+4=13$
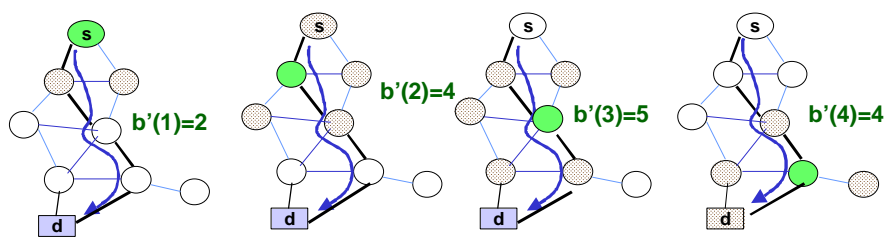

Figure 2. Blocking metric of an alternative route $B(k)=2+4+5+4=15$

We show the interference-aware route construction algorithm in Figure 3. Beginning with a single Mesh BS node, we add one SS into the mesh at a time. The time sequence of node $\eta$ joining the mesh is represented by $\sigma(\eta)$. When the SS node $\eta$ joins the mesh, it will select the Sponsoring Node with the minimum blocking.

In the interference-aware route construction scheme, the blocking metric information is incorporated into the Network Descriptor of a MSH-NCFG message. When a new node is scanning for active network during the network entry process, the new node chooses the potential Sponsoring Node based on the blocking metric information to reduce the interference of the multihop route and hence to improve the throughput.

$$
\begin{aligned}
& \mathrm{S} \leftarrow\{0\} \quad / / \text { node } 0 \text { is the root node; Initialize the set of selected nodes } \\
& \mathrm{N}_{\mathrm{S}} \leftarrow\{1,2, \ldots, n\} \quad / / \text { Initialize the set of unselected nodes } \\
& p(i) \leftarrow \varnothing, \quad i \in\{1,2, \ldots, n\} \quad / / \text { Initialize parent node for node } 1,2, \ldots, \mathrm{n} \\
& \text { Do if } \mathrm{N}_{\mathrm{S}} \neq \varnothing \\
& \eta \leftarrow \underset{i \in N_{S} \cap \text { Neighbor }(S)}{\arg \max } \sigma(i) \text { //Node } \eta \text { with high } \sigma(\eta) \text { value joins first } \\
& \mathrm{C}(\eta) \leftarrow \operatorname{Neighbor}(\eta) / / \text { All nodes within transmission distance of } \eta \\
& W(\eta) \leftarrow C(\eta) \bigcap S \quad \text { //Candidate parent nodes of node } \eta \\
& p(\eta) \leftarrow \underset{i \in W(\eta)}{\arg \min } B(i) \quad / / \text { Select the node with minimum blocking }
\end{aligned}
$$

Figure 3. Interference-Aware Route Construction Algorithm

\section{B. Interference-Aware Scheduling}

The design goal of the proposed interference-aware scheduling is to exploit concurrent transmission opportunity to achieve high spectral utilization and hence high system throughput. The interference-aware scheduling seeks to maximize the number of concurrent transmissions, without creating exceeding interference for other simultaneous transmission. This is achieved by taking into the consideration the traffic capacity request of each SS. We denote the capacity request of an SS node $k$ from the Mesh BS as $D(k)$. The Mesh BS grants radio resource according to the Application Layer capacity requests, $D(k)-s$, of all SS nodes and the route information of the mesh network.

We show the interference-aware scheduling algorithm in Figure 4 . With the obtained route information from network entry and the initialization process, the node capacity request $D(k)$ can also be equivalently represented in terms of link demands $Y(j)$ for every link $\mathrm{j}$. The scheduling algorithm iteratively determines ActiveLink $(t)$, which is the set of active links at the time $t$. In each allocation iteration $t$, a link with the highest unallocated traffic demand is selected for next allocation of a unit traffic. The scheduling algorithm is designed to find the maximum number of concurrent transmissions. To satisfy the SINR constraints of concurrent transmissions, the Blocked_Neighbor( $k$ ) function is used to exclude interfering links that are located in the neighborhood of $k$. The iterative allocation continues until there is no unallocated capacity request. 


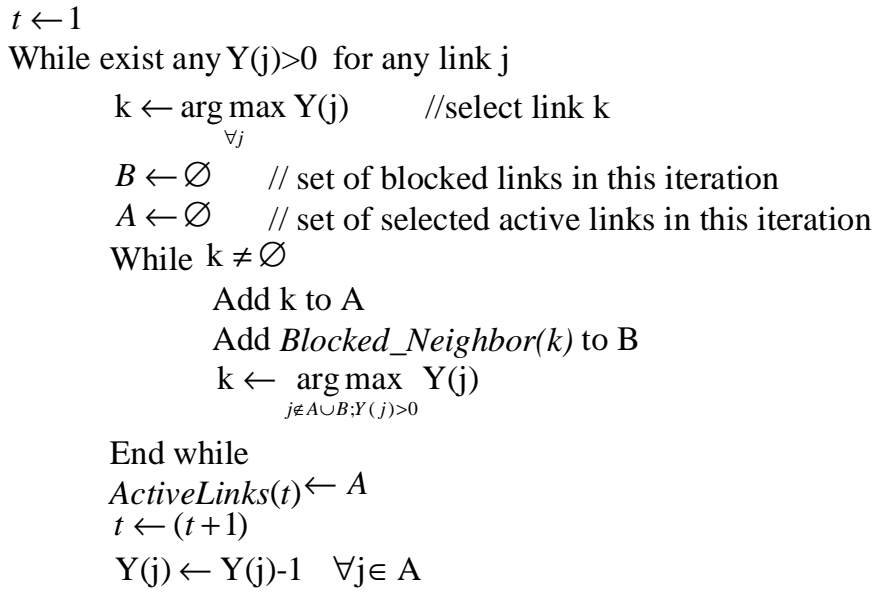

End while

Figure 4. Interference-Aware Scheduling Algorithm

\section{PERFRORMANCE EVALUATION}

We have evaluated the system throughput of IEEE 802.16 mesh in two scenarios: a linear chain topology and a random mesh topology. The proposed schemes are compared to the "no spatial reuse MSH-CSCH" scheduling example given in IEEE 802.16a standard [1], which we refer to in this paper as the basic scheme. To see how the 802.16 mesh schemes perform, we also investigate a theoretical upper-bound based on linear programming. The network throughput of the basic 802.16 scheme and the proposed interference-aware scheme are simulated on the Matlab platform.

\section{A. Optimal Solution From Linear Program}

A linear programming algorithm is formulated to model the network throughput upper bound of IEEE 802.16 mesh networks. Similar to the modeling technique demonstrated in [5], the network activity is modeled in terms of the normalized time fraction as a real number between 0 and 1 . The set $S$ represents all the possible transmission schemes in terms of link bandwidth $R$ (bits/second). In a given transmission scheme $\mathrm{j}$ with active transmitting node $t$ and receiving nodes $r, S(t, j)$ is set to $-R(t, r)$, the link bandwidth between the node pair $(\mathrm{t}, \mathrm{r})$, if node $t$ is transmitting to node $r$, and $S(r, j)$ is set to $+R(t, r)$. All other $S(x, j)$ are set to 0 if node $x$ is neither transmitting nor receiving in the $j$-th transmission scheme. In a transmission scheme, multiple concurrent transmissions are allowed, if all receiving conditions are satisfied. The variable $x(j)$ represents the normalized time fraction that the $j$-th transmission scheme is active in unit time. At all receivers, it is required that $\operatorname{SINR}(\mathrm{j})>\gamma$, which is the minimum reception SINR threshold.

The objective function of this linear programming algorithm is to maximize the overall network throughput. A resource allocation constraint is imposed to ensure fair throughput allocation among users. End-to-end throughput is proportionally allocated based on the parameter $L$; i.e., user $\alpha$ will be allocated the end-to-end throughput proportional to $L(\alpha)$. In addition, the summation of all transmission time fractions $x(j)$ should be less than unit time.
The optimal solutions of the linear programming algorithm, is used as a performance comparison benchmark for the proposed interference-aware 802.16 mesh network scheme. Since the optimal linear programming algorithm is computed based on arbitrarily slicing of time fractions of all feasible transmission combinations, the performance of the discretetime event driven design of proposed schemes is capped by the performance of the linear programming algorithm. Thus the performance of the linear programming algorithm serves as an upper bound.

Maximize $\sum_{i>1} \sum_{\forall j} x(j) S(i, j)$

Such that

$$
\begin{aligned}
& L(\alpha) / \sum_{\forall j} x(j) S(\alpha, j)=L(\beta) / \sum_{\forall j} x(j) S(\beta, j), \forall \alpha, \beta \in\{1,2, \ldots, N\} \\
& \sum_{\forall j} x(j) \leq 1, j \in\{1,2, \ldots, M\} \\
& 0 \leq x(j) \leq 1
\end{aligned}
$$

Figure 5. The Linear Programming Algorithm

\section{B. Throughput Performance in Chain Topology}

The first scenario considers only the scheduling problem in a chain topology IEEE 802.16 multihop network. In this chain topology, route construction is straightforward; i.e., data packets are always forwarded along the chain. Here, we investigate the effectiveness of interference-aware scheduling. The throughput of the basic 802.16 mesh network without interference-aware scheduling is compared to that of the proposed scheme and to the upper bound obtained from the linear programming algorithm. As shown in Figure 6, the proposed interference-aware scheduling scheme performance approaches the upper bound, while outperforms the basic 802.16 mesh mode significantly.

Also as shown in the figure, the number of nodes in this multihop 802.16 network affects the possibility of concurrent transmissions and hence adversely affects the network throughput. In the basic 802.16 mesh scheme, the network throughput drops significantly as the number of nodes increases, because of the limited spatial reuse. On the other hand, with the interference-aware concurrent transmission, the normalized overall throughput degrades significantly less as the number of nodes increases.

The proposed interference-aware scheduling scheme is more scalable than the basic scheme. As the length of relay route increases with the number of nodes in the network, the overall network throughput decreases due to the fact that a packet needs to be forwarded several times. If the increase in the degree of spatial reuse is less than the increase of number of hops, the network throughput will decrease. Depending on the number of relay hops and the network topology, there are limitations on the degree of spatial reuse that could be achieved. By comparing the results of the optimal linear programming algorithm, we concluded that our proposed 
scheme could achieve a near-optimal network throughput and spatial utilization.

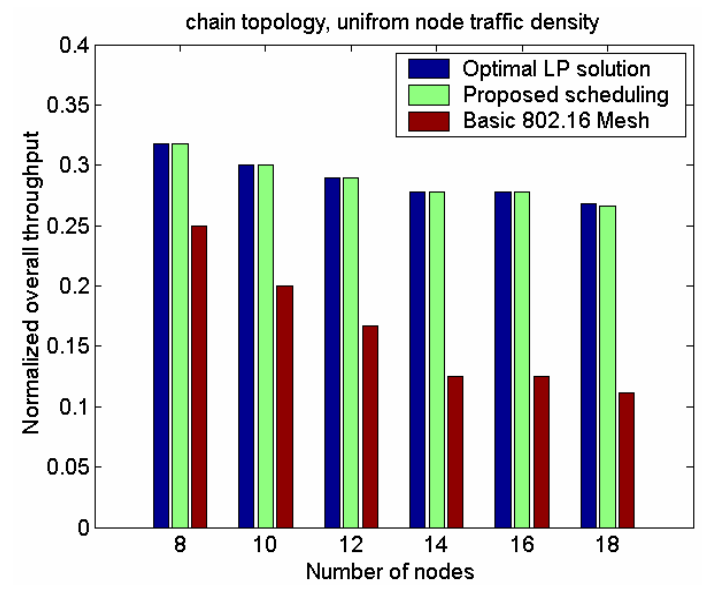

Figure 6. Overall throuhgput of a chain topology 802.16 network

\section{Throughput Performance in Random Topology}

In the second scenario, we considered both scheduling and routing in a random-topology 802.16 mesh network. Locations of a set of mesh nodes are randomly generated. The order of nodes joining the mesh is not correlated with the nodes' locations, but is randomly determined. The mesh formation begins with the Mesh BS node. Then the SS nodes join one-byone. Any node that has already joined the mesh network could become a Sponsoring Node. When a new node joins the existing mesh network, depending on the number of candidate SNs within the new node's transmission range, it may hear multiple MSH-NCFG advertisement messages.

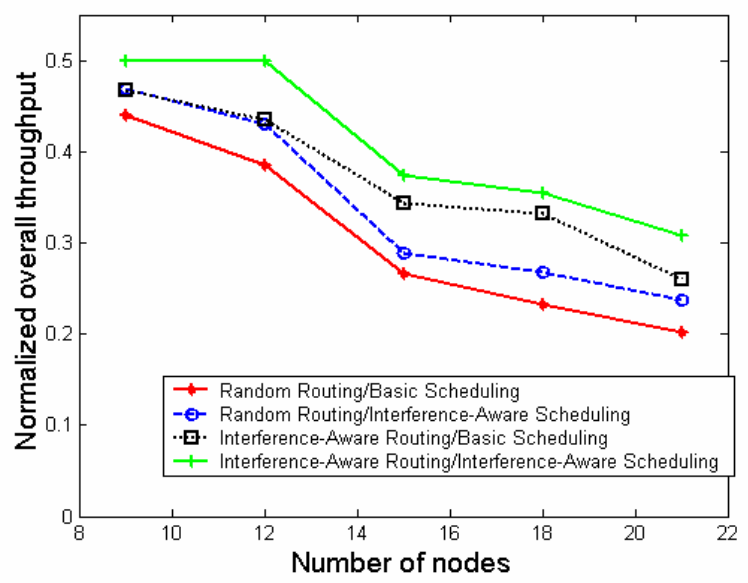

Figure 7. Throuhgput performance of a random topology 802.16 mesh

In the interference-aware routing scheme, the new node selects the Sponsoring Node as the candidate SN with the minimum blocking metric. For comparison, the new node randomly selects a Sponsoring Node in the random routing scheme. This type of route construction is denoted as random routing in Figure 7. Both, the proposed interference-aware scheduling and the basic 802.16 mesh scheduling were simulated. Similar to the chain topology scenario, we discuss the overall network throughput decreases as the number of nodes increases. As shown in Figure 7, the scheme with both, the interference-aware routing and the scheduling achieves the highest network throughput. The "interference-aware routing only" scheme has the second best performance. The "interference-aware scheduling only" scheme also outperforms the basic 802.16 mesh scheme. As a result, we conclude that the proposed interference-aware framework could effectively enhance the basic IEEE 802.16 Mesh mode operation. In the design process of scheduling and route construction in mesh network, one should adopt the interference-aware design concept and hence exploit the benefit of concurrent transmissions with less interference. Consequently, the spectral utilization in mesh networks is enhanced with less interference and more spatial reuse.

\section{ConClusions}

Allowing concurrent transmissions to achieve high spatial reuse is essential for scalable wireless mesh network design. We proposed an interference-aware research framework for the emerging IEEE 802.16 Mesh mode to improve spectral utilization. Using this framework, we introduced an interference-aware route construction algorithm for 802.16 mesh network initialization process to improve the network throughput by selecting routes with minimal interference to existing nodes. In addition, a load-aware and interferenceaware scheduling algorithm for centralized scheduling in IEEE 802.16 Mesh mode is also discussed. Simulation results show that the proposed schemes effectively improve the network throughput performance in IEEE 802.16 mesh networks and achieve high spectral utilization.

\section{REFERENCES}

[1] IEEE Std 802.16a-2003, "IEEE Standard for Local and metropolitan area networks--Part 16: Air Interface for Fixed Broadband Wireless Access Systems--Amendment 2: Medium Access Control Modifications and Additional Physical Layer Specifications for 2-11 GHz," 2003

[2] IEEE Std 802.16-2004 (Revision of IEEE Std 802.16-2001), "IEEE Standard for Local and Metropolitan Area Networks Part 16: Air Interface for Fixed Broadband Wireless Access Systems," 2004

[3] M. Conti, G. Maselli, G. Turi, and S. Giordano, "Cross-layering in mobile ad hoc network design," IEEE Computer, vol. 37, pp. 48-51, 2004.

[4] S. Shakkottai, T. S. Rappaport, and P. C. Karlsson, "Cross-layer design for wireless networks," IEEE Communications Magazine, vol. 41, pp. $74-80,2003$

[5] S. Toumpis and A.J. Goldsmith, "Capacity Regions For Wireless Ad hoc Networks," IEEE International Conference on Communications (ICC), 2002 